\title{
On the Analytical Mechanics of Chemical Reactions. Classical Mechanics of Linear Collisions*
}

\author{
R. A. Marcus \\ Noyes Chemical Laboratory, University of Illinois, Urbana, Illinois
}

(Received 3 June 1966)

\begin{abstract}
The classical mechanics of chemically reactive linear collisions is investigated for vibrationally nearadiabatic reactions. A coordinate system which passes smoothly from one suited to the reactants to one suited to the products is used. The Hamilton-Jacobi equation is then solved in the adiabatic approximation by introduction of an "adiabatic-separable" method. Nonadiabatic corrections, which describe the probability of vibrational transitions, are also calculated. They involve the Fourier component of local internal centrifugal and vibration frequency-change terms. The reaction coordinate for the adiabatic system is shown to be that curve on which local vibrational and internal centrifugal forces balance pointwise. Applications can be made to the role of translational-vibrational energy interchange in reactions, reaction-crosssection theory, bobsled effect, and other topics. The results may be compared with electronic computer calculations as they become available.
\end{abstract}

\section{INTRODUCTION}

$\mathbf{I}^{\mathrm{N}}$ $\mathrm{N}$ the present paper we consider the dynamical aspects of motion of a system on a potential-energy surface for chemical reaction in a linear collision, $\mathrm{A}+\mathrm{BC} \rightarrow \mathrm{AB}+\mathrm{C}$. In a center-of-mass system there are only two coordinates, but the problem does not permit separation of variables for actual surfaces. The present paper is a companion to one recently submitted on the quantum mechanics of these systems ${ }^{1}$ and the introduction to that paper applies to the present one also.

In this reaction the vibrational mode is originally a $\mathrm{BC}$ vibration, then becomes a symmetric stretching $\mathrm{ABC}^{\ddagger}$ vibration and, finally, an $\mathrm{AB}$ vibration. ${ }^{2}$ This vibrational mode is adiabatic under certain circumstances, particularly when the system does not strike the curved part of the " reaction path" with too high a speed. ${ }^{3}$ We consider such reactions and calculate also nonadiabatic corrections. They contain a Fourier component of a local centrifugal term and of a term depending on vibration-frequency variation. The component is shown to involve

$$
\exp \left(i \int \omega d t\right)
$$

rather than $\exp (i \omega t)$, because of the variation in vibration frequency during the motion.

Implications of these results for the characterization of the reaction coordinate in chemical reactions, for translational-vibrational energy transfer, the bobsled effect, and vibrationally nonadiabatic "locking in" of energy in reaction-cross-section calculations are con-

\footnotetext{
* Supported by a grant from the National Science Foundation.

1 R. A. Marcus, J. Chem. Phys. 45, 4493 (1966), preceding paper.

${ }^{2}$ This change in nature of the vibration $\left(\mathrm{AB}\right.$ to $\mathrm{ABC}^{\ddagger}$ to $\left.\mathrm{BC}\right)$ is seen from the potential-energy contour plots, such as those in Ref. 3.

${ }^{3}$ Reactions such as $\mathrm{H}+\mathrm{H}_{2} \rightarrow \mathrm{H}_{2}+\mathrm{H}$ appear to be vibrationally adiabatic at low to moderate relative translational velocities. For analyses of exact electronic computer results on these reactions, see Footnote 8 of Ref. 1.
}

sidered. Implications for tunneling calculations have been considered elsewhere. ${ }^{1}$

\section{HAMILTONIAN AND COORDINATE SYSTEMS}

The potential-energy surface for these collision problems, plotted using skewed coordinate axes ${ }^{4}$ in a center-of-mass coordinate system, can be represented by well-known contour diagrams. ${ }^{5}$ The surface possesses two valleys, one corresponding to configurations of initial reactants and the other corresponding to those of final products. The valleys are frequently separated by some saddle-point region, a "col." In the chemicalreaction case the system surmounts the barrier, but in the vibrational energy transfer problem ${ }^{6}$ it lacks the energy to do so and returns to the valley, perhaps with some change in vibrational energy.

The path of minimum potential energy from a valley floor to the top of the pass and then of descent to the floor of the other valley is a useful one for describing the potential-energy function for the surface. The corresponding curve in the coordinate space (a projection of the path onto that space) is called the "reaction path" to distinguish it from the "reaction coordinate" which is described later. It is a curved path in the interaction region and a rectilinear path in the region of separated reactants or separated products. Motion perpendicular to it is vibrational.

We introduce a coordinate system ${ }^{8}$ suited to the

${ }^{4}$ The axes are skewed to remove cross terms from the kineticenergy expression in center-of-mass Cartesian coordinates. Because of this diagonalization the system can be represented in its motion by that of a single point of mass $\mu .^{5 a}$ Equation (1) of the present paper is based partly on this fact.

5 (a) S. Glasstone, K. J. Laidler, and H. Eyring, The Theory of Rate Processes (McGraw-Hill Book Co., Inc., New York, 1941), Chap. 3; (b) R. E. Weston, J. Chem. Phys. 31, 892 (1959).

${ }^{6}$ When the energy is insufficient to cause reaction, the present treatment becomes a method for treating the vibration-translation energy exchange problem.

7 H. S. Johnston, Advan. Chem. Phys. 3, 131 (1961).

${ }^{8}$ H. C. Corben and P. Stehle, Classical Mechanics (John Wiley \& Sons, Inc., New York, 1960), 2nd ed., pp. $319 f f$. 
surface topography, as follows. Let $C$ be any curve in the coordinate space. It could be taken as the "reaction path," but when greater accuracy is desired it can be chosen in a different way, described later.

The distance from any point in the space to the nearest point on the curve is $x$. The length along $C$ to that point on $C$ is $s$. Thus, any point in the space can be described by coordinates $x$ and $s$. (Ambiguities could occur if $C$ had loops etc.) The kinetic energy $T$ may be expressed in terms of the momenta $p_{x}$ and $p_{s}$ conjugate to $x$ and $s$, respectively, ${ }^{8}$

$$
T=\left(p_{x}^{2} / 2 \mu\right)+p_{s}^{2} /\left[2 \mu(1+\kappa x)^{2}\right],
$$

where $\kappa$ is the curvature of $C$ at the point corresponding to $(x, s)$ and $\mu$ is the effective mass of moving point representing the dynamical system ${ }^{4} ; \mu$ is unity in a mass-weighted space, but is often chosen to be the reduced mass of the reactants in the chemical-reaction case. The final answer is independent of this choice, of course, since it merely determines the units of the coordinates.

The potential energy $V$ is

$$
\begin{aligned}
V & =V_{1}(s)+V_{2}(x, s), \\
V_{2}(0, s) & =0,
\end{aligned}
$$

where $V_{1}(s)$ is the potential energy along Curve $C$ and $V_{2}(x, s)$ is defined by (2) and (3). When $C$ is chosen to be the "reaction path" $V_{2}(x, s)$ is a vibrational Morse-like potential-energy function of $x$ and may depend weakly on $s$.

\section{ADIABATIC SOLUTION}

The Hamilton-Jacobi ${ }^{9}$ equation obtained from (1) and (2), rewritten slightly, is

$$
\begin{aligned}
& \left(\frac{1}{2 \mu(1+\kappa x)^{2}}\right)\left[\left(\frac{\partial W}{\partial s}\right)^{2}+2 \mu V_{1}(s)\right] \\
& +\left(\frac{1}{2 \mu}\right)\left(\frac{\partial W}{\partial x}\right)^{2}+U_{2}(x, s)=\alpha_{1},
\end{aligned}
$$

where

$$
U_{2}(x, s)=\left[1-(1+\kappa x)^{-2}\right] V_{1}(s)+V_{2}(x, s) ;
$$

$\alpha_{1}$ is the total energy, and $W(x, s, \alpha)$ is Hamilton's characteristic function. It is a generating function for a canonical transformation,

$$
p_{i}=\partial W / \partial q_{i}, \quad Q_{i}=\partial W / \partial \alpha_{i},
$$

where the $p_{i}$ and $q_{i}$ are the old momenta $\left(p_{x}, p_{s}\right)$ and coordinates $(x, s)$, respectively. The new momenta are constants of the motion $\alpha_{i}$. The $Q_{i}$ are the new coordinates.

${ }^{9} \mathrm{H}$. Goldstein, Classical Mechanics (Addison-Wesley Publ. Co., Inc., Reading, Mass., 1950), pp. 280f.
For certain surfaces the function $U_{2}(x, s)$ depends but weakly on $s,{ }^{10}$ and we can then write (7) as a zeroth-order solution,

$$
W \cong W_{1}(s, \alpha)+W_{2}(x, \alpha) .
$$

From (5) one then obtains

$$
\begin{aligned}
& (2 \mu)^{-1}\left(d W_{1} / d s\right)^{2}+V_{1}(s) \\
& =\left[-(2 \mu)^{-1}\left(d W_{2} / d x\right)^{2}+\alpha_{1}-U_{2}(x, s)\right]\left[(1+\kappa x)^{2}\right] .
\end{aligned}
$$

The left side is independent of $x$. If the right side were independent of $s$, both sides could be set equal to a constant, $\alpha_{2}$. However, the right side depends weakly on $s$, and so such an $\alpha_{2}$ is now a slowly varying function of $s$, yielding an adiabatic approximation:

$$
\begin{gathered}
(2 \mu)^{-1}\left(d W_{1} / d s\right)^{2}+V_{1}(s)=\alpha_{2}(s), \\
(2 \mu)^{-1}\left(d W_{2} / d x\right)^{2}+U_{2}(x, s)+\left[\alpha_{2} /(1+\kappa x)^{2}\right]=\alpha_{1} .
\end{gathered}
$$

One sees from (9) with $p_{s}=d W_{1} / d s$ that $\alpha_{2}$ serves as a slowly varying energy for the angular motion, referred to Curve $C$. The weak dependence of $\alpha_{2}$ on $s$ when $C$ is chosen to coincide with the "reaction coordinate curve" is shown later by Eq. (18).

It is convenient to define a function $U_{3}(x, s)$ which acts as an effective potential energy for the vibrational motion:

$$
U_{3}(x, s)=U_{2}(x, s)+\left[\alpha_{2} /(1+\kappa x)^{2}\right] .
$$

The minimum of $U_{3}$ occurs at $x=x_{0}$. Thus ${ }^{11}$ (definition of $x_{0}$ ),

$$
\partial V_{2}\left(x_{0}, s\right) / \partial x_{0}=\left(\alpha_{2}-V_{1}\right) 2 \kappa /\left(1+\kappa x_{0}\right)^{3} .
$$

The curve $x=x_{0}(s)$ is the "reaction coordinate," often mentioned in chemical kinetics though not previously defined throughout the space. ${ }^{12}$ When $C$ is chosen to be the reaction path, the functions $V_{1}(s)$ and $V_{2}(x, s)$ are very simply expressed. A better

${ }^{10}$ For some surfaces $U_{2}(x, s)$ minus a function of $s$ depends but weakly on $s$. This (initially unknown) function is then added and subtracted in Eq. (4). It is then present as an additional term in both sides of Eq. (8). When both sides of this modified (8) are set equal to a parameter weakly dependent on $s$, the cited additional term can be absorbed in the latter, again yielding Eqs. (9) and (10). There are of course some reactions which are markedly vibrationally nonadiabatic and for these Eqs. (9) and (10) are inapplicable.

${ }_{11}$ Throughout this paper, $\partial V_{2}\left(x_{0}, s\right) / \partial x_{0}$ and $\partial^{2} V_{2}\left(x_{0}, s\right) / \partial x_{0}{ }^{2}$ denote the values of $\partial V_{2}(x, s) / \partial x$ and $\partial^{2} V_{2}(x, s) / \partial x^{2}$ at $x=x_{0}$.

${ }^{12}$ The term "reaction coordinate" is used in the common way as that curve along which the vibrationally averaged system moves (strictly speaking, in the harmonic-oscillator approximation) on going from reactants' region of the space through activated complex's to the products' one. The current justification of activatedcomplex theory [J. O. Hirschfelder and E. Wigner, J. Chem. Phys. 7, 616 (1939); M. A. Eliason and J. O. Hirschfelder, ibid. 30, 1426 (1959); L. Hofacker, Z. Naturforsch. 18a, 607 (1963)] is tied to an adiabatic hypothesis for the motion of all coordinates but the reaction coordinate. In this respect, the definition of "reaction coordinate" should be tied to a vibrationally adiabatic reaction, as in the present paper. The "reaction coordinate" defined in the first sentence is of course a member of a family of curves. When $C$ is taken to be the "reaction coordinate" these curves are: $x=$ constant. 
choice for $C$ for some purposes, however, is that curve for which $x_{0}(s)=0$. C then becomes the "reaction coordinate" itself and, according to (12) is that curve which satisfies (13):

and

$$
\partial V_{2}\left(x_{0}, s\right) / \partial x_{0}=\left(\alpha_{2}-V_{1}\right) 2 \kappa
$$

$$
x_{0}=0 .
$$

Examination of (9) and (13) reveals that $-\partial V_{2} / \partial x_{0}$ is the local vibrational force and that the right side, which equals $\kappa p_{s}{ }^{2} / \mu$, is the local centrifugal force. (It is $\mu \dot{s}^{2} / R$, where $R$ is the radius of curvature of $C, 1 / \kappa$, at this point $s$, and where the dot denotes $d / d t$.)

It is also instructive to rewrite (8) with the aid of (6) and (11):

$$
\begin{array}{r}
{\left[(2 \mu)^{-1}\left(d W_{2} / d x\right)^{2}+U_{3}(x, s)-U_{3}\left(x_{0}, s\right)\right]+V_{1}(s)} \\
+V_{2}\left(x_{0}, s\right)+\left[p_{8}{ }^{2} / 2 \mu\left(1+\kappa x_{0}\right)^{2}\right]=\alpha_{1} .
\end{array}
$$

The term in brackets is the local vibrational energy at $s$. The sum of the next two is the potential energy on the "reaction coordinate" at $s$, since $V_{1}(s)$ is the potential energy on the "reaction path" $C$ and $V_{2}\left(x_{0}, s\right)$ is the increment needed to go from $C$ to the "reaction coordinate" at the same $s$. The last term in (14) is the kinetic energy of the $s$ motion along the reactioncoordinate curve. The sum of these terms is the total energy, $\alpha_{1}$.

In the vibrationally adiabatic approximation the vibrational action variable $J$ is constant during the motion, ${ }^{13}$ just as the vibrational quantum number is constant in a quantum-mechanical treatment of such reactions. The local vibrational energy is denoted by $E\left(J_{0}, s\right){ }_{14}^{14}$ where $J_{0}$ is the initial value of $J$. We obtain

$(2 \mu)^{-1}\left(d W_{2} / d x\right)^{2}+U_{3}(x, s)-U_{3}\left(x_{0}, s\right)=E\left(J_{0}, s\right)$.

An equation for $\alpha_{2}$ is obtained from (9), (14), and (15):

$\alpha_{2}=\alpha_{1}-E\left(J_{0}, s\right)-V_{2}\left(x_{0}, s\right)+\left[1-\left(1+\kappa x_{0}\right)^{-2}\right]\left(\alpha_{2}-V_{1}\right)$.

Equations (12) and (16) can be solved by various means for $\alpha_{2}(s)$ and $x_{0}(s)$. After obtaining a solution for $\alpha_{2}(s)$ and $x_{0}(s)$, Eq. (9) is next solved: Since $p_{s}$ equals $d W_{1} / d s$ [Eqs. (6) and (7)] and equals $\mu \dot{s}(1+$ $\left.\kappa x_{0}\right)$, as seen from Hamilton's equation, $\dot{s}=\partial H / \partial p_{s}$, the dependence of $s$ on $t$ is obtained from

$$
\int_{s 0}^{s} \frac{\mu^{\frac{1}{3}}\left(1+\kappa x_{0}\right) d s}{\left[2\left(\alpha_{2}-V_{1}\right)\right]^{\frac{1}{3}}}=t-t_{0}
$$

where $s_{0}$ is the value of $s$ at $t=t_{0}$, and so depends on the origin chosen for $s$.

\footnotetext{
13 For example, D. Ter Haar, Elements of Hamiltonian Mechanics (North-Holland Publ. Co., Amsterdam, 1961), Chap. 6.

14 With the use of angle-action variables for the vibration the energy depends only on one of them, the action. (Compare Refs. 8 or 13 .)
}

An adiabatic solution of the problem has thereby been obtained. When $C$ is chosen to be the reactioncoordinate curve, $x_{0}=0$, and Eqs. (16) and (17) become

$$
\begin{gathered}
\alpha_{2}=\alpha_{1}-E\left(J_{0}, s\right), \\
\mu^{\frac{1}{s}} \int_{s_{0}}^{s} \frac{d s}{\left[2\left(\alpha_{2}-V_{1}\right)\right]^{\frac{1}{3}}}=t-t_{0} .
\end{gathered}
$$

\section{HARMONIC-OSCILLATOR APPROXIMATION}

When the harmonic-oscillator approximation is used for the effective potential-energy function (11) in Eq. (10), Eqs. (15), (16), and (18) apply as before with $^{15}$

where

$$
E\left(J_{0}, s\right)=J_{0 \omega_{2}} / 2 \pi
$$

$$
\mu \omega_{2}^{2}=\left(\partial^{2} V_{2} / \partial x_{0}^{2}\right)+\left[6\left(\alpha_{2}-V_{1}\right) \kappa^{2} /\left(1+\kappa x_{0}\right)^{4}\right] .
$$

When the reaction path is chosen as Curve $C$, $\partial^{2} V_{2}\left(x_{0}, s\right) / \partial x_{0}^{2}$ equals $\omega^{2}$, where $\omega / 2 \pi$ is the "natural" frequency of vibration at $s$, i.e., the actual frequency if centrifugal forces were not considered:

$(C=$ reaction path $) \quad \omega_{2}{ }^{2}=\omega^{2}+6\left(\alpha_{2}-V_{1}\right) \kappa^{2} / \mu\left(1+\kappa x_{0}\right)^{4}$.

When the reaction coordinate is chosen as Curve $C$, $x_{0}=0$ in (21).

\section{NONADIABATIC CORRECTION}

For the following arguments, Curve $C$ is taken to be the reaction path, and the harmonic-oscillator approximation for $V_{2}(x, s)$ is used. One way of estimating the nonadiabatic correction is as follows.

Hamilton's equations for $p_{x}$ and for $\dot{x}$, applied to the original Hamiltonian, yield

$$
\mu \ddot{x}+\partial V_{2} / \partial x=\kappa p_{k}^{2} / \mu(1+\kappa x)^{3} .
$$

On adding $-\ddot{x}_{0}$ to both sides of this equation, introducing the adiabatic result for $p_{a}{ }^{2}$ on the right side, ${ }^{16}$ and expanding the right side and $\partial V_{2} / \partial x$ about $x=x_{0}$, retaining terms in $\left(x-x_{0}\right)$, one finds

$$
\ddot{x}-\ddot{x}_{0}+\omega_{2}{ }^{2}\left(x-x_{0}\right)=-\ddot{x}_{0} .
$$

Because of the dependence of $\omega_{2}$ on time, through the dependence of $s$ and $p_{s}$ on time according to (17),

${ }^{15}$ Reference 8, p. 190, Eq. (62.7) yields (20).

${ }_{16}$ Logically, this step could have been delayed until post (30), so that the latter, at least, would be exact in this respect. Instead of the right side of (24) one would have

$$
\begin{aligned}
-\ddot{x}_{0}+\left[\kappa p_{\mathrm{s}}^{2} / \mu^{2}(1+\right. & \left.\left.\kappa x_{0}\right)^{3}\right]-\left(\omega^{2} x_{0} / \mu\right) \\
& \times\left\{3 \kappa^{2}\left[p_{\mathrm{s}}^{2}-2 \mu\left(\alpha_{2}-V_{1}\right)\right] /\left(1+\kappa x_{0}\right)^{4} / \mu^{2}\right\}\left(x-x_{0}\right) .
\end{aligned}
$$

However, the step was made here to abbreviate the notation, since the results after (30) are the same. 
we introduce a reduced time scale $\tau$ and a new variable $y^{17}$ :

$$
\begin{aligned}
& \tau=\int_{t^{\prime}=t_{0}}^{t} \frac{\omega_{2}}{\omega_{0}} d t^{\prime}+t_{0}, \\
& y=\left(\omega_{2} / \omega_{0}\right)^{\frac{1}{2}}\left(x-x_{0}\right),
\end{aligned}
$$

where $\omega_{2}$ is a function of time $t^{\prime}$ in (25) and $\omega_{0}$ is the value of $\omega_{2}$ at $t=t_{0}$. The time $t=t_{0}$ is to be chosen to correspond to the system's being in the form of reactants.

From (25) one now obtains after some manipulation

where

$$
\left(d^{2} y / d \tau^{2}\right)+\omega_{0}^{2} y=-\left(\omega_{0} / \omega_{2}\right)^{\frac{1}{x_{0}}}+F y,
$$

$$
F=\left(\omega_{0} / \omega_{2}\right)^{\frac{1}{2}}\left(d^{2} / d \tau^{2}\right)\left(\omega_{2} / \omega_{0}\right)^{\frac{1}{2}}
$$

and where the dot denotes $d / d t$, as before.

Equation (27) is equivalent to the integral equation (29), as substitution of the latter into the former shows:

$y=\frac{A}{\omega_{0}^{\frac{1}{2}}} \sin \left(\omega_{0} \tau+\delta\right)+\frac{1}{\omega_{0}} \int_{-\infty}^{\tau} \sin \omega_{0}\left(\tau-\tau^{\prime}\right) G\left(\tau^{\prime}\right) d \tau^{\prime}$,

where the lower limit of $t=t_{0}$ was replaced by $-\infty$, since $G$ vanishes for $t<t_{0} . A$ and $\delta$ are constants. $G(\tau)$ is the right side of (27), expressed in terms of the reduced time variable,

$$
G(\tau) \equiv-\left(\omega_{0} / \omega_{2}\right)^{3} \ddot{x}_{0}+F y .
$$

Introduction of the adiabatic approximation into the right side of Eq. (29) for $y$ yields a nonadiabatic correction: the first term on the right side is the adiabatic result and the second is the nonadiabatic one.

At infinite time, integration of (29) twice by parts yields

$y=\frac{A}{\omega_{0}^{\frac{1}{3}}} \sin \left(\omega_{0} \tau+\delta\right)+\frac{1}{\omega_{0}} \int_{-\infty}^{\infty} \sin \omega_{0}\left(\tau-\tau^{\prime}\right) G^{*}\left(\tau^{\prime}\right) d \tau^{\prime}$,

where

$$
G^{*}(\tau) \equiv \omega_{0}^{2} x_{0}\left(\omega_{2} / \omega_{0}\right)^{\frac{1}{2}}+x\left(d^{2} / d \tau^{2}\right)\left(\omega_{2} / \omega_{0}\right)^{\frac{1}{2}} .
$$

The second term in (32) is expected to be negligible usually.

The nonadiabatic correction is useful in at least three respects.

(1) In the case of chemical reaction it yields the vibrational-energy change, in excess of that expected from adiabatic considerations, $J_{0}\left(\omega_{\infty}-\omega_{0}\right) / 2 \pi$, where $\omega_{\infty}$ in the value of $\omega$ at $t=\infty$.

(2) In the case of translation-vibrational energy transfer it yields the vibrational energy change.

(3) In chemical reaction it yields the nonadiabatic correction to calculations of the reaction threshold in the two-dimensional case and of the "nonavailable

\footnotetext{
${ }^{17}$ A similar transformation is used for a related (but homogeneous) equation, e.g., A. Erdelyi, Asymptotic Expressions (Dover Publications, Inc., New York, 1956), p. 79.
}

energy" in the actual ( $n$-dimensional) case. (The "nonavailable energy" is the energy unavailable for distribution among other coordinates when $\dot{s}=0$ at the saddle point. It plays a role in a statistical-dynamical theory of reaction cross sections. ${ }^{18}$ )

\section{CHEMICAL REACTION}

In the region of the separated products, the path $C$ is rectilinear, and so $\kappa=x_{0}=0$. Let $\omega=\omega_{\infty}$ in this valley. The final vibrational energy is $\frac{1}{2} \mu \omega_{\infty}^{2} x^{2}+\frac{1}{2} \mu \dot{x}^{2}$. For the change of vibrational energy $\Delta E_{\mathrm{vib}}$ we thus obtain

$$
\begin{aligned}
\Delta E_{\mathrm{vib}}=\frac{\left(\omega_{\infty}-\omega_{0}\right) J_{0}}{2 \pi} & +\left(\frac{J_{0} \mu \omega_{\infty}^{2}}{\omega_{0} \pi}\right)^{\frac{1}{2}} \\
& \times \int_{-\infty}^{\infty} \cos \left(\omega_{0} \tau^{\prime}+\delta\right) G^{*}\left(\tau^{\prime}\right) d \tau^{\prime} \\
& +\frac{1}{2} \mu \frac{\omega_{\infty}}{\omega_{0}}\left|\int_{-\infty}^{\infty} \exp \left(i \omega_{0} \tau^{\prime}\right) G^{*}\left(\tau^{\prime}\right) d \tau^{\prime}\right|^{2} .
\end{aligned}
$$

\section{VIBRATION-TRANSLATION ENERGY TRANSFER}

When the system returns to its original valley without being able to surmount the reaction barrier, $\omega_{\infty}$ is $\omega_{0}$ and we obtain (34) for the increase of vibrational energy,

$$
\begin{aligned}
\Delta E_{\mathrm{vib}}=\left(\frac{J_{0} \mu \omega_{0}}{\pi}\right)^{\frac{1}{2}} \int_{-\infty}^{\infty} \cos \left(\omega_{0} \tau^{\prime}+\delta\right) G^{*}\left(\tau^{\prime}\right) d \tau^{\prime} \\
+\frac{\mu}{2}\left|\int_{-\infty}^{\infty} \exp \left(i \omega_{0} \tau^{\prime}\right) G^{*}\left(\tau^{\prime}\right) d \tau^{\prime}\right|^{2} .
\end{aligned}
$$

\section{NONAVAILABLE ENERGY AND REACTION THRESHOLD}

In the two-dimensional case, the $\alpha_{1}$ in (4) is a constant. In applications to the $n$-dimensional one $\alpha_{1}$ may vary with $s$, because of energy loss to other coordinates, the form of $\alpha_{1}(s)$ depending on the model used.

When $\dot{s}$ vanishes at the top of the barrier, $x_{0}$ also vanishes there. The total energy at that point then equals the potential energy there and the vibrational energy, $E_{\mathrm{vib}} . E_{\mathrm{vib}}$ equals $\frac{1}{2} \mu\left(\dot{x}-\dot{x}_{0}\right)^{2}+\frac{1}{2} \mu \omega_{2}^{2}\left(x-x_{0}\right)^{2}$, where now $\omega_{2}=\omega$ at this point. ${ }^{19}$ From (29) one finds

$$
\begin{array}{r}
E_{\mathrm{vib}}=\frac{J_{0 \omega}}{2 \pi}+\left(\frac{J_{0 \mu \omega^{2}}}{\omega_{0} \pi}\right)^{\frac{1}{2}} \int_{-\infty}^{\tau} \cos \left(\omega_{0} \tau^{\prime}+\delta\right) G\left(\tau^{\prime}\right) d \tau^{\prime} \\
+\quad+\frac{1}{2} \mu \frac{\omega}{\omega_{0}}\left|\int_{-\infty}^{\tau} \exp \left(i \omega_{0} \tau^{\prime}\right) G\left(\tau^{\prime}\right) d \tau^{\prime}\right|^{2},
\end{array}
$$

where $r$ is the reduced time to reach the top of the barrier.

\footnotetext{
${ }^{18}$ R. A. Marcus, J. Chem. Phys. 45, 2630 (1966).

${ }^{19}$ According to (22), $\omega_{2}$ equals $\omega$ when $\alpha_{2}=V_{1}$. According to (9), $\alpha_{2}$ equals $V_{1}$ when $p_{\mathrm{a}}$ vanishes, i.e., when $\dot{s}$ vanishes. That is, $\omega_{2}$ equals $\omega$ for the cited conditions.
} 
Let $s^{*}$ denote the value of $s$ at the top of the barrier. When $x_{0}$ is a symmetric function of $s-s^{*}, \dot{x}_{0}$ vanishes at $s=s^{*}$. Upon integrating (35) twice by parts one now obtains (35) but with $G$ replaced by $G^{*}$.

The threshold energy for the reaction in the twodimensional case, $\Delta E$, is

$$
\Delta E=V_{1}\left(s^{*}\right)+E_{\mathrm{vib}}-\left(J_{0 \omega_{0}} / 2 \pi\right),
$$

where $E_{\text {vib }}$ is given by (35). The second term in (35) yields the "nonadiabatic leak," mentioned elsewhere ${ }^{18}$ as a way of accounting for the small but finite cross sections observed in numerical integrations for classical collisions at energies below "threshold."

\section{BOBSLED EFFECT}

As the system proceeds around the curved part of the valley bottom, i.e., the region where the "reaction path" is curved in coordinate space, it experiences the expected centrifugal force. This result is exhibited by
Eq. (12), according to which $x_{0}(s)$ is positive when $p_{s}{ }^{2}$ is positive. ${ }^{20}$ This effect is the bobsled effect noted by Wall and Porter ${ }^{21}$ in their numerical calculations: A bobsled going downhill goes partly up the valley wall when the "reaction path" is curved.

If the downhill velocity in the latter problem is sufficiently great when the bobsled mounts the curved portion, it will go up the valley wall as far as it can go, then down across the valley bottom, up the other wall, and so on. That is, when the velocity is sufficiently high while the system reaches the curved part of the "reaction path," oscillations are induced where there were none present originally. This effect is an example of vibrational nonadiabaticity and is exhibited by Eq. (33) with $J_{0}=0$.

${ }^{20}$ For example, if $p_{\mathrm{s}}{ }^{2}$ is positive, $\alpha_{2}-V_{1}$ is positive [Eq. (9) ]. In that case, $\partial V_{2} / \partial x_{0}$ is positive [Eq. (12)]. This result implies that $x_{0}(s) \geq 0$. (For example, in the harmonic-oscillator approximation, $\partial V_{2} / \partial x_{0}$ is $\mu \omega^{2} x_{0}$.) $x_{0}(s)=0$ when $\kappa=0$.

${ }^{21}$ F. T. Wall and R. N. Porter, J. Chem. Phys. 39, 3112 (1963).

\title{
Spectra of Praseodymium in Yttrium Gallium Garnet and in Yttrium Aluminum Garnet
}

\author{
F. N. HOOGE \\ Philips Research Laboratories, N. V. Philips' Gloeilampenfabrieken, Eindhoven, The Netherlands
}

(Received 11 July 1966)

\begin{abstract}
The absorption and fluorescence spectra of $\mathrm{Pr}^{8+}$ in $\mathrm{YGaG}$ and in YAlG were measured at temperatures between $16^{\circ} \mathrm{K}$ and room temperature. Crystal-field calculations, using a cubic field with $A_{4}^{0}=-350 \mathrm{~cm}^{-1}$ and $A_{6}{ }^{0}=90 \mathrm{~cm}^{-1}$, gave a reasonable fit with the experimentally found energy levels. Some manifolds overlap due to the large crystal-field splitting. The centers of gravity found by fitting the calculated manifolds to the experimental data are close to and well in line with the centers for $\mathrm{Pr}^{3+}$ in $\mathrm{LaCl}_{3}$ and $\mathrm{LaBr}_{3}$.
\end{abstract}

\section{INTRODUCTION}

$\mathbf{A}^{1}$ FEW years ago Wong, Stafsudd, and Johnston ${ }^{1}$ published the spectra of $\mathrm{Pr}^{3+}$ in different lattices. According to them one of these lattices was YAlG, but in fact it must have been $Y G a G$, since there is good agreement between their experimental results and our YGaG spectra. From our spectra we determined the energy levels in the manifolds, and we were more optimistic than Wong et al. about the possibility of applying crystal-field calculations. 'To determine the centers of gravity, even to estimate them very roughly, it is necessary to make this calculation because the splitting of the manifolds is very wide, and some manifolds overlap.

The large number of lines in the transitions between two manifolds clearly demonstrates that the site symmetry at the $\mathrm{Pr}^{3+}$ ion is low. Nevertheless we use a cubic field in our approximation, and we then assume

${ }^{1}$ E. Y. Wong, O. M. Stafsudd, and D. R. Johnston, J. Chem. Phys. 39, 786 (1963). that the noncubic terms will give only a relatively small splitting of the terms that are degenerate in a cubic field. If this assumption is correct the small noncubic splittings can help to distinguish between cubic $A, E$, and $T$ levels. The ${ }^{1} D_{2}$ manifold at 17000 $\mathrm{cm}^{-1}$ provides a good example of our approach. See Fig. 1.

We use a cubic field for the following reasons:

(1) The $\mathrm{Pr}^{3+}$ ion is surrounded by eight oxygen atoms at the corners of a distorted cube. ${ }^{2,3}$

(2) For a cubic field we can use the energy diagrams of Lea, Leask, and Wolf. ${ }^{4}$

(3) We could not find all levels in each manifold. Complicated crystal-field potentials with many adjustable parameters can therefore be fitted in many

${ }^{2}$ S. Geller and M. A. Gilleo, J. Phys. Chem. Solids 3, 30 (1957). ${ }^{3} M$. T. Hutchings and W. P. Wolf, J. Chem. Phys. 41, 617 (1964).

${ }^{4}$ K. R. Lea, M. J. M. Leask, and W. P. Wolf, J. Phys. Chem. Solids 23, 1381 (1962). 